\title{
Conditioned inhibition in feature negative discrimination learning with pigeons
}

\author{
DANIEL S. GOKEY and ROBERT L. COLLINS \\ Florida State University, Tallahassee, Florida 32306
}

\begin{abstract}
Discrimination performance was investigated with pigeons using feature negative (FN) discrimination procedures which differed in the temporal arrangement of the stimuli on Strials. In both procedures, a single common element was presented on reinforced $(\mathrm{S}+)$ trials. In the simultaneous $\mathrm{FN}$ procedure, a distinguishing element was presented simultaneously with the common element of $\mathrm{S}-$ trials. In the sequential $\mathrm{FN}$ procedure, the distinguishing element preceded onset of the common element on $\mathbf{S}-$ trials. In two experiments, the sequential FN procedure yielded better discrimination performance. In Experiment 1, a summation test designed to separate learning and performance variables indicated that sequential FN subjects had learned the negative relationship between the distinguishing element and reinforcement while simultaneous FN subjects had not. In Experiment 2, summation and acquisition tests indicated that the distinguishing element developed inhibitory properties in the sequential FN procedure but not in the simultaneous FN procedure.
\end{abstract}

Jenkins and Sainsbury $(1969,1970)$ found that pigeons failed to respond differentially on reinforced $(\mathrm{S}+)$ and nonreinforced $(\mathrm{S}-)$ trials in what was termed a "feature negative" (FN) discrimination. In that procedure, $\mathrm{S}+$ trials were signaled by a visual stimulus display (A) and $S$ - trials were signaled by the same display with an added "distinguishing element" (i.e., AB). Subsequent research has shown that the temporal relationship between $\mathrm{A}$ and $\mathrm{B}$ on $\mathrm{S}$ - trials is a major determinant of whether or not pigeons differentially respond on $\mathrm{S}+$ and $\mathrm{S}-$ trials in FN discriminations. The discrimination is acquired when $\mathrm{A}$ and $\mathbf{B}$ are presented sequentially (i.e., $B \rightarrow A$; Hearst, 1978; Collins, Note 1) or in rapid alternation (Farthing, 1971), but not when they are presented simultaneously as in the Jenkins and Sainsbury experiments.

In both the simultaneous and sequential FN procedures, B is negatively correlated with reinforcement and is always presented in conjunction with an otherwise reinforced simulus, $\mathrm{A}$. This procedure is similar to the paradigm described by Pavlov (1927) for establishing $\mathrm{B}$ as a conditioned inhibitor. Thus, the differential acquisition of the discrimination suggests a differential development of $\mathbf{B}$ as a conditioned inhibitor in the two cases. The following experiments inves-

This research was supported in part by NIMH Grants MH 11218 and $\mathrm{MH}$ 30487-01 (R. L. Collins, principal investigator) and by NSF Grant BNS-121346226 (M. E. Rashotte, principal investigator). These experiments are based on a thesis by Daniel $\mathrm{S}$. Gokey which fulfilled part of the requirements for the MS degree at Florida State University, December 1977. The authors wish to thank Michael E. Rashotte for his helpful comments on an earlier draft of this paper. R. L. Collins is now at Georgia Institute of Technology. Reprints may be obtained from Daniel S. Gokey, Department of Psychology, Florida State University, Tallahassee, Florida 32306. tigated whether the discrimination between $\mathrm{S}+$ and $\mathrm{S}-$ trials in the sequential and simultaneous FN procedures is related to the differential development of conditioned inhibition or to some other mechanism of response reduction.

\section{EXPERIMENT 1}

In Experiment 1, pigeons were trained with a simultaneous FN or a sequential FN procedure. After training, all subjects received a one-session test in extinction which consisted of three types of trials: $A$ alone $(A)$, the simultaneous presentation of $A$ and $B(A B)$, and the sequential presentation of $A$ and $B$ $(B \rightarrow A)$. During the test, two comparisons were made for each subject, responding in the simultaneous compound relative to $\mathrm{A}$ alone and responding in the sequential compound relative to $\mathrm{A}$ alone.

The design of the test phase was similar to one common test for inhibition, a summation test. In a summation test, a known excitor is presented alone and in conjunction with the supposed inhibitor. If the stimulus in inhibitory, it will suppress responding to the excitor (e.g., Cornell \& Strub, 1965; Rescorla \& LoLordo, 1965). In the present experiment, if $B$ were to become a conditioned inhibitor in the sequential FN procedure, then it should suppress responding to $A$ in both the simultaneous and the sequential test compound; if B were to fail to develop inhibitory properties in the simultaneous FN procedure, it should suppress responding to $\mathrm{A}$ in neither test compound.

Pigeons trained with either FN procedure, however, may learn the negative relationship between $B$ and reinforcement, but, as suggested by Jenkins (1973), $\mathrm{B}$ fails to suppress responding to $\mathrm{A}$ when the two 
are simultaneously compounded. This implies that the temporal relationship of the stimulus elements (a performance variable), rather than something learned, may be responsible for the differential ability of $\mathrm{B}$ to suppress responding in the two FN procedures. With the use of two compounds in the summation test, the experimental design also constituted a modification of the crossover design that has been used previously to separate learning and performance variables (e.g., Grant \& Schneider, 1948, 1949). In the design used here, the effects of the two types of test compounds were compared within each group of subjects. If the differential discrimination performance during $\mathrm{FN}$ training was the result of a performance variable, then both simultaneous $\mathrm{FN}$ and sequential FN subjects should suppress responding to $A$ in the sequential test compound but not in the simultaneous test compound.

\section{Method}

\section{Subjects}

Twelve experimentally naive white Carneaux pigeons served as subjects. They were maintained at approximately $80 \%$ of their ad-lib weight for the duration of the experiment and were individually housed in wire-mesh cages under a 12-h light-dark cycle. All sessions occurred during the light portion of the cycle. Water and grit were constantly available in the home cages.

\begin{abstract}
Apparatus
Four identical plastic and metal test chambers were used. The chambers, which measured $38.7 \times 39.4 \times 35.9 \mathrm{~cm}$, were painted flat black and were enclosed in slightly larger wooden boxes. Three response keys, $2.5 \mathrm{~cm}$ in diameter, were located on one wall of the chamber. The keys were spaced $8.3 \mathrm{~cm}$ center to center and were $26.7 \mathrm{~cm}$ above the floor. The keys were illuminated by applying $28 \mathrm{~V}$ ac to one of three No. 1820 bulbs housed behind each key and were covered by red, white, or green lenses. Only the left and center keys were used in the experiment. Grain was presented by raising a hopper to a $5 \times 5 \mathrm{~cm}$ opening beneath the middle key. The lower edge of the opening was $8.9 \mathrm{~cm}$ above the floor, which was illuminated whenever the hopper was raised.

While sessions were in progress, the chamber was illuminated by a $110 \mathrm{~V}$ ac $25-\mathrm{W}$ bulb located behind an opaque Plexiglas plate in the center of the ceiling. White noise $\left(80 \mathrm{~dB}\right.$ re $\left.20 \mu \mathrm{N} / \mathrm{m}^{2}\right)$ masked extraneous sounds, and an exhaust fan provided ventilation. Electromechanical control and recording equipment were located in an adjacent room.
\end{abstract}

\section{Procedure}

Magazine training. Magazine training was given for a minimum of 2 days and continued until all pigeons ate reliably and consistently when the hopper was presented for 4-sec periods at variable intervals averaging $30 \mathrm{sec}$.

Training. The pigeons were assigned to two groups of six, designated "simultaneous FN" (Sim) and "sequential FN" (Seq). As described below, these group designations refer to the temporal relation between the $A$ and $B$ stimuli presented on $S-$ trials. Each group received 15 training sessions consisting of $20 \mathrm{~S}+$ trials and $20 \mathrm{~S}-$ trials, with a variable intertrial interval averaging $60 \mathrm{sec}$ (Fleshler \& Hoffman, 1962). No more than three $\mathrm{S}+$ or $\mathbf{S}-$ trials occurred successively. The $A$ and $B$ stimuli were 6-sec red or white keylights, counterbalanced across subjects in each group.

On S + trials, A was presented on one of the two keys and was followed by $4-\mathrm{sec}$ access to grain in an autoshaping (Brown \& Jenkins, 1968) procedure. The unused key remained dark throughout $\mathrm{S}+$ trials.
On S- trials, for Group Sirn, the A and B stimuli were presented simultaneously, one stimulus on each response key. For Group Seq, however, B was presented for $6 \mathrm{sec}$ on one key, followed immediately by $A$ on the other key. For both groups. $A$ was assigned to the left key and B to the center key on a random half of the $S-$ trials.

Summation test. On the day following the 15 th discrimination training session, all subjects received a single 60 -trial test in which three types of nonreinforced trials occurred. The $A$ stimulus was presented alone on 20 trials, which were identical to $S+$ trials in discrimination training except that reinforcement was omitted. The A and B stimuli were presented simultaneously on 20 trials that were identical to the S- trials of Group Sim. The A and B stimuli were presented sequentially on 20 trials that were identical to the S- trials of Group Seq. Four of each type of trial occurred in an irregular order during successive blocks of 12 test trials. The A stimulus occurred equally often on the left and center keys when it was presented alone. On trials when A and B were presented in compound, the stimuli were also counterbalanced across key location.

Dependent variables and statistical analysis. During both the training phase and the summation test, rates of keypecking (responses/minute) to the A and B stimuli were determined in each session. In the summation test, rates were computed for the entire test session and for individual blocks of 12 trials. Nonparametric statistical analyses were used to assess between-group and withingroup differences. In all cases, one-tailed tests were employed.

\section{Results}

The performance of Groups Seq and Sim in each of the 15 training sessions is shown in Figure 1. Responding to $\mathrm{A}$ on $\mathrm{S}+$ trials was rapidly acquired and remained high for both groups throughout training. The mean rate of responding to $\mathrm{A}$ on $\mathrm{S}+$ trials did not differ between the groups for the last 5 days of training (Wilcoxon rank sum test, $W=45, p>.05$ ). Responding to $B$ showed an initial increase, especially in Group Seq, but dropped out almost completely after only a few sessions in both groups. The rate of responding to A was comparable on $\mathbf{S}+$ and $\mathbf{S}-$ trials for both groups in the initial six sessions, but, for Group Seq only, responding to A on S- trials subsequently fell below the level maintained to $A$ on $\mathrm{S}+$ trials. Over the last 5 days of training, the mean rate of responding to A was 83.5 and 53.8 responses/ min on S + and S- trials, respectively, for Group Seq.

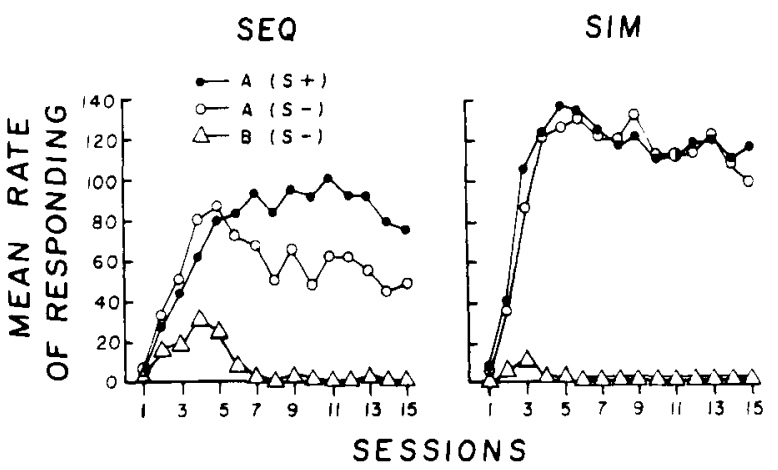

Figure 1. Training data for Groups Seq and Sim in Experiment 1. The mean rates of responding (per minute) to the individual stimulus elements are shown for the 15 training sessions (see text for details). 
This difference was statistically reliable (Wilcoxon signed rank test, $\mathrm{T}=0, \mathrm{p}<.05$ ).

\section{Summation Test}

The data for the 60-trial summation test are shown in Figure 2. Responding was comparably maintained across test blocks for both groups, except for one subject in Group Seq whose data have been excluded for failing to respond for the entire test session.

The remaining five subjects of Group Seq (left panel) responded significantly less to $A$ in sequential or simultaneous compound relative to $\mathrm{A}$ alone (Wilcoxon signed rank tests, Ts $=0$, ps $<.05$ ). There was no difference between responding to $A$ in sequential compound relative to $A$ in simultaneous compound (Wilcoxon signed rank test, $\mathrm{T}=1.5, \mathrm{p}>.05$ ). For Group Sim (right panel), there was no suppression of responding to $\mathrm{A}$ when it was presented in either simultaneous or sequential compound with B relative to $A$ alone (Wilcoxon signed rank tests, $\mathrm{Ts} \geqslant 2.5$, ps $>.05)$.

\section{Discussion}

The training data of this experiment replicated other findings that sequential presentation of stimuli on Strials in an FN discrimination with pigeons yields differential responding to $\mathrm{S}+$ and $\mathrm{S}-$, whereas simultaneous presentations of the stimuli fail to do so (Hearst, 1978; Collins, Note 1).

New data on the source of the between-group difference was provided by the summation test. Group Seq suppressed responding to $A$ in both simultaneous and sequential compounds; information provided by $B$ was used to suppress responding to A regardless of the temporal arrangement of the compound elements. In contrast, Group Sim failed to suppress responding
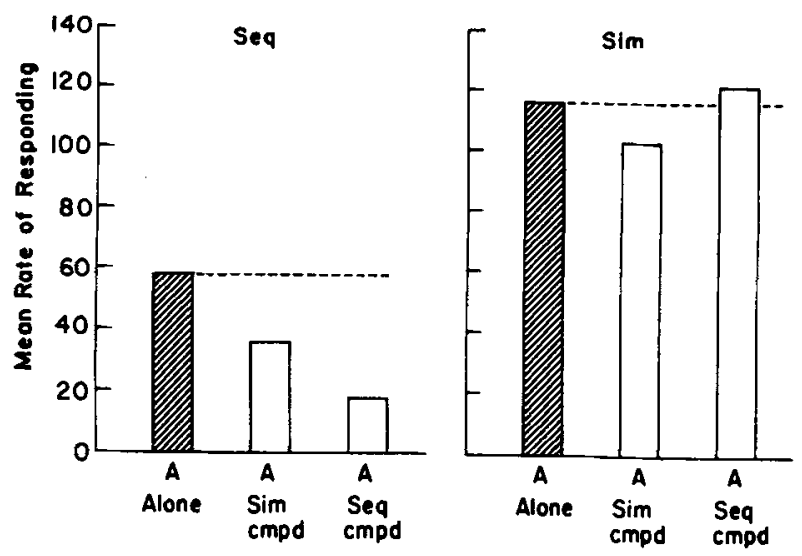

STIMULUS

Figure 2. Summation test data for Groups Seq and Sim in Experiment 1. The mean rates of responding (per minute) to $A$ in simultaneous $(A B)$ and sequential $(B \rightarrow A)$ compound test trials relative to $A$-alone trials are shown for the entire (60-trial) test session (see text for details). to $A$ in either test compound. These subjects were unable to suppress responding to $A$ in a situation (i.e., a sequential compound) known to allow B to suppress responding. Thus, because the summation test resembled a crossover test, these data indicated that differential responding on $\mathbf{S}+$ and $\mathbf{S}-$ trials is related to what is learned about $B$ during training and is not related to a performance variable. Sequential FN subjects learned the negative relationship between $B$ and reinforcement, while simultaneous FN subjects failed to do so. Furthermore, these summation test data provide evidence that $B$ developed inhibitory properties in sequential FN subjects but did not do so in simultaneous FN subjects. To evaluate conclusively the inhibitory status of $\mathrm{B}$ as a result of FN training, however, requires a more complete test of inhibition.

\section{EXPERIMENT 2}

Experiment 2 provided a more complete assessment of the inhibitory status of the B stimulus in the sequential and simultaneous FN procedures. It also extended discrimination training to determine whether additional training would lead to acquisition of the discrimination in simultaneous FN subjects. In particular, training continued for 200 trials more than in Experiment 1, and B was tested in "acquisition" and "summation" tests whose joint use is recommended to evaluate whether or not a stimulus is inhibitory (Hearst 1972; Rescorla, 1969). In an acquisition test, a neutral stimulus and the supposed inhibitory stimulus are converted into conditioned excitors through pairings with a US. An inhibitory stimulus will require more pairings than the neutral stimulus to reach a criterion of acquisition. As described previously, in a summation test the putative inhibitor is presented in combination with an excitatory stimulus. An inhibitor will suppress responding to the excitor. The summation test in Experiment 2 was identical to that of Experiment 1, except that, in the present experiment, the excitatory stimulus used during the test was a newly conditioned stimulus, $C$ rather than the A stimulus used during training. Thus, if B were inhibitory, it should suppress responding to $\mathrm{C}$.

\section{Subjects and Apparatus}

\section{Method}

Thirty-two experimentally naive White Carneaux pigeons were housed and maintained under the conditions described in Experiment 1 and were trained in the same apparatus employed there.

\section{Procedure}

The 32 subjects were magazine trained and were then assigned to either a simultaneous $F N$ procedure $(N=16)$ or the sequential FN procedure $(\mathrm{N}=16)$ employed in Experiment 1. All aspects of training were identical to the first experiment, except that discrimination training continued for 20 sessions instead of 15 sessions. At the completion of training, sequential FN subjects were as- 
signed to two groups designated "Seq-Sum" and "Seq-Acq," and simultaneous FN subjects were assigned to two groups designated Sim-Sum" and "Sim-Acq." "Sum" (summation) and "Acq" (acquisition) refer to the type of test the group received following training. These tests are described below.

Summation test. Groups Seq-Sum and Sim-Sum received one session in which a novel stimulus (a 6-sec green keylight, designated the $C$ stimulus) was paired with 4-sec access to grain in an autoshaping procedure. Forty pairings were given in this session, with an average intertrial interval of $60 \mathrm{sec}$. The $C$ stimulus was presented in an irregular sequence on the left and center keys, occurring on each key on half the trials in the session.

On the day following acquisition to $C$, these subjects received six $S+$ and six $S-$ trials in the sequential or simultaneous FN procedure appropriate for their training assignment. They then received a summation test in which there were five 12-trial blocks (60 trials), each block comprising four nonreinforced presentations of $\mathrm{C}$ alone, $\mathrm{B}$ and $\mathrm{C}$ presented simultaneously, and B and C presented sequentially. The summation test was identical to that employed in Experiment 1, except that $C$ was used as the excitatory stimulus, rather than $\mathrm{A}$.

Acquisition test. Groups Seq-Acq and Sim-Acq received an acquisition test in which the $B$ stimulus from $S-$ trials in discrimination training and novel stimulus, $C$ (a 6-sec green keylight), were paired with $4-\mathrm{sec}$ access to grain in an autoshaping procedure on 20 trials in each session. The $B$ and $C$ stimuli were presented equally often on the left and center keys. Acquisition sessions continued for each subject until it met a criterion of sustained responding on each stimulus. The criterion was defined as three out of four successive presentations of the stimulus with at least one keypeck.

\section{Results}

The left panel of Figure 3 shows that sequential FN subjects discriminated between $\mathrm{S}+$ and $\mathrm{S}-$ trials. Beginning with Session 7 , and over the remaining training sessions, responding to $A$ on $S-$ trials was significantly less than responding to $\mathbf{A}$ on $\mathbf{S}+$ trials (normal theory approximation for large-sample Wilcoxon signed rank test, $\mathrm{T}^{*}=3.36, \mathrm{p}<.01$ ). The right panel of Figure 3 shows that simultaneous FN subjects began to discriminate around Session 16. Over the last 5 days of training, the reduction in responding to $A$ on $S-$ relative to $A$ on $S+$ trials was significant (normal theory approximation for largesample Wilcoxon signed rank test, $\mathrm{T}^{*}=2.89$, $\mathrm{p}<$ $.01)$. Though both groups eventually responded differentially on $\mathrm{S}+$ and $\mathrm{S}$ - trials, the performance of

\section{SEQUENTIAL FN}

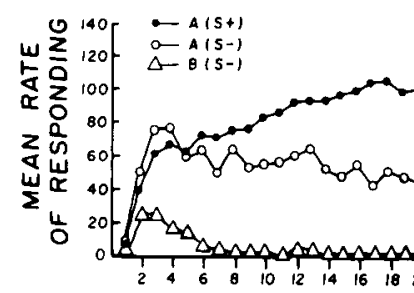

SESSIONS

Figure 3. Training data for sequential FN and simultaneous FN subjects in Experiment 2. The mean rate of responding (per minute) to the individual stimulus elements are shown for the $\mathbf{2 0}$ training sessions (see text for details).

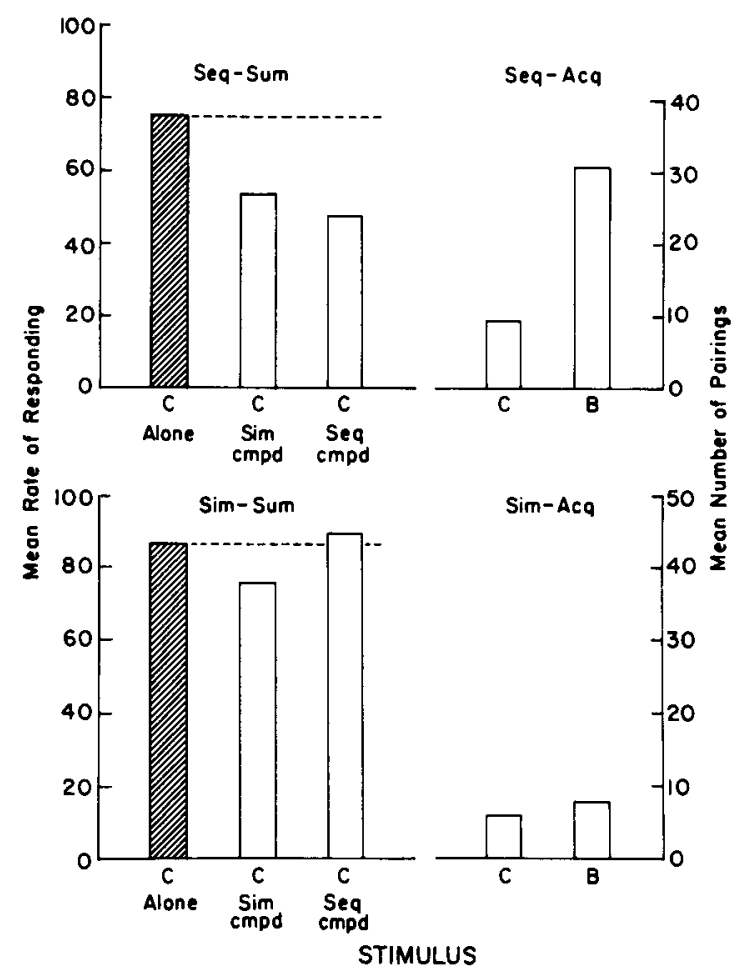

Figure 4. Inhibition test data for sequential and FN simultaneous FN subjects in Experiment 2. Summation test data (left panels) show the mean rates of responding (per minute) to $C$ in the simultaneous $(B C)$ and Sequential $(B \rightarrow C)$ compound test trials relative to $\mathrm{C}$-alone trials for the first two blocks ( 24 trials) of the test session. Acquisition test data (right panels) show the mean number of pairings to the criterion of acquisition for both $B$ and C stimuli (see text for details).

sequential FN subjects was superior to that of simultaneous FN subjects. Normal theory approximation for large-sample Wilcoxon rank sum tests indicated no difference between groups in responding to $A$ on $\mathrm{S}+$ trials $\left(\mathrm{W}^{*}=1.36, \mathrm{p}>.05\right)$, but significantly lower responding to A on $\mathrm{S}-$ trials for sequential FN subjects than for simultaneous FN subjects over the last 5 days of training $\left(\mathrm{W}^{*}=2.67, \mathrm{p}<.01\right)$.

\section{Summation Test}

In the session prior to the summation test, both Group Seq-Sum and Group Sim-Sum acquired responding to $\mathrm{C}$. Keypecking to $\mathrm{C}$ was readily acquired by both groups and, once acquired, responding was maintained.

The summation test data shown in the left panel of Figure 4 are based on only the first two blocks (24 trials) of the test session because the overall rates of responding to $\mathrm{C}$ declined rapidly in the later blocks. This was probably due to the small amount of excitatory conditioning of $\mathrm{C}$ prior to the test. For Group Seq-Sum (upper left panel), responding was significantly suppressed to $C$ in both sequential and simultaneous test compounds relative to $\mathrm{C}$ alone 
(Wilcoxon signed rank test, $\mathrm{T}=3, \mathrm{p}<.05, \mathrm{~T}=1$, $\mathrm{p}<.01$, respectively). There was no difference between responding to $C$ in simultaneous compound relative to $C$ in sequential compound by these subjects (Wilcoxon signed rank test, $\mathrm{T}=16.5, \mathrm{p}>.05$ ). For Group Sim-Sum (lower left panel), responding to $C$ was suppressed in simultaneous compound, but was slightly enhanced in the sequential compound relative to $\mathrm{C}$ alone. Wilcoxon signed rank tests indicated that the reduction of responding to $C$ in simultaneous compound was significant $(T=3, p<.05)$, but there was no difference in the rate of responding to $C$ in sequential compound relative to $\mathrm{C}$ alone $(\mathrm{T}=28, \mathrm{p}>.05)$.

\section{Acquisition Test}

The acquisition test data are shown in the right panels of Figure 4. Group Sim-Acq acquired responding equally rapidly to $\mathrm{B}$ and $\mathrm{C}$ (Wilcoxon signed rank test, $\mathrm{T}=21.5, \mathrm{p}>.05$ ). For Group Seq-Acq, however, significantly more pairings were required to reach the criterion of acquisition to $B$ than of acquisition to $\mathrm{C}$ (Wilcoxon signed rank test, $\mathrm{T}=36, \mathrm{p}<.01$ ).

\section{Discussion}

As in Experiment 1, the discrimination performance of subjects trained with the sequential FN procedure showed more rapid and more complete development of the discrimination between $\mathrm{S}+$ and $\mathrm{S}$ - trials than subjects trained with the simultaneous $\mathrm{FN}$ procedure. Thus, the presence of $\mathrm{B}$ more effectively controlled suppressed responding to the compound relative to $A$ alone when $B$ was presented in a sequential rather than in a simultaneous compound. Unlike Experiment 1, there was some evidence that training with the simultaneous FN procedure did not preclude the acquisition of discrimination between $\mathrm{S}+$ and $\mathrm{S}-$ trials. Simultaneous FN subjects suppressed responding to A on S- trials relative to A on S + trials, but not until after about 16 training sessions.

After discrimination training, two tests were used to determine whether $\mathrm{B}$ had become a conditioned inhibitor in either group. In the summation test, sequential FN subjects suppressed responding when B was presented in simultaneous or sequential compound with C. Simultaneous FN subjects suppressed responding to $\mathrm{C}$ when $\mathrm{B}$ was presented in simultaneous compound. This result suggests that $B$ may have become inhibitory during simultaneous FN training. This interpretation, however, must be tempered, because these subject failed to suppress responding to $\mathrm{C}$ in sequential compound trials. If $B$ was inhibitory, it should suppress responding in the compound regardless of the temporal arrangement of the stimulus elements. In the acquisition test, acquisition of keypecking to $B$ was retarded relative to $C$ in sequential FN subjects, but not in simultaneous FN subjects. Taken together, the results of these tests indicate that $\mathrm{B}$ had clearly become a conditioned inhibitor in the sequential FN procedure but, on the whole, not in the simultaneous FN procedure.

\section{GENERAL DISCUSSION}

Two major points were established in these experiments. First, the difference in the acquisition of a discrimination between the sequential FN and simultaneous FN procedures is due to a difference in what is learned about the stimulus element (B) which distinguishes $S+$ from $S-$ trials. Sequential FN subjects learned to a greater extent that $B$ was negatively correlated with reinforcement than did subjects trained with the simultaneous FN procedure. Second, the difference in what was learned about $B$ resulted in a difference in the development of $\mathrm{B}$ as a conditioned inhibitor. Taken together, the results of the summation test and acquisition test of Experiment 2 indicated that $\mathrm{B}$ developed conditioned inhibition in the sequential FN procedure, but failed to do so in the simultaneous FN procedure. Learning the discrimination in the simultaneous FN procedure, then can be attributed to a difficulty in the development of $\mathrm{B}$ as a conditioned inhibitor.

These data are relevant to an interpretation by Mackintosh (1969) for the failure of simultaneous FN subjects to discriminate between $\mathrm{S}+$ and $\mathrm{S}-$ stimuli. He suggests that the failure to discriminate can be attributed to orientation effects caused by the use of discrete localized, visual stimuli. Thus, responding to one stimulus prevents orienting toward the other stimulus and subjects fail to learn about B because they are orienting toward $A$. This interpretation also may account for the difference in learning about $B$ between the simultaneous FN and sequential FN procedures. In the sequential FN procedure, orientation toward and learning about $B$ is facilitated because $A$ is not present to interfere with orientation toward B. However, data from the summation tests seem to be inconsistent with Mackintosh's interpretation. If orienting toward A prevents orienting toward $B$, then sequential FN subjects should not be able to use information previously learned about B to suppress responding in simultaneous compound trials. This, however, was not the case; sequential FN subjects suppressed responding in simultaneous compound trials. Thus, pigeons do more than "look where they peck," as Mackintosh (1969) suggested.

An extension of Mackintosh's interpretation, however, might account for the difference in discrimination performance between these two $\mathrm{FN}$ procedures. In the simultaneous FN procedure, with both $\mathrm{A}$ and $\mathrm{B}$ present on $S$ - trials, these subjects must either orient toward A or B, in exclusion of the other, or divide orientation and learning between the two stimuli. In the sequential FN procedure, this competition for orientation does not exist. $\mathrm{B}$ is presented alone prior to $A$, so the subject may orient solely toward B. 
With this interpretation, sequential FN subjects, because of their greater opportunity in the absence of $A$ to orient toward and learn about $\mathrm{B}$, should acquire the discrimination between $\mathrm{S}+$ and $\mathrm{S}-$ trials more rapidly than simultaneous FN subjects. Also, unless the subject orients solely toward A on $\mathrm{S}-$ trials, at some point in simultaneous FN training, the bits of time spent orienting toward $B$ should accrue sufficiently for the learned inhibitory effect of B to suppress responding to $\mathbf{A}$ in compound. Amount of training, then, is crucial in determining whether or not B develops inhibitory properties in the simultaneous FN procedure. In the present experiments, no simultaneous FN subject had acquired the discrimination when training was terminated after 15 sessions. When training was terminated after 20 sessions, however, simultaneous FN subjects had acquired the discrimination to some extent and evidence that $\mathrm{B}$ might be developing inhibitory properties was provided by the summation test; Group Sim-Sum subjects suppressed responding to $\mathrm{C}$ in simultaneous compound test trials. Perhaps, if training were extended beyond 20 sessions, discrimination performance might continue to improve and both summation and acquisition tests would indicate that $B$ had become a conditioned inhibitor.

This interpretation is not intended as a general model for the development of conditioned inhibition. It is designed to account for the differential development of conditioned inhibition in the sequential FN and the simultaneous $\mathrm{FN}$ procedures using discrete localized, visual stimuli. Whether this interpretation can be extended into other areas using different types of stimuli remains to be determined.

\section{REFERENCE NOTE}

1. Collins, R. L. Successive discrimination between a single element and a sequential compound stimulus. Paper presented at the annual meeting of the Southeastern Psychological Association, New Orleans, 1976.

\section{REFERENCES}

Brown, P. L., \& Jenkins, H. M. Auto-shaping of the pigeon's keypeck. Journal of the Experimental Analysis of Behavior, $1968,11,1-8$.

Cornell, J. M., \& Strub, H. M. A technique for demonstrating the inhibition function of $S^{\star}$. Psychonomic Science, 1965, 3, 25-26.

Farthing, G. W. Discrimination of compound stimuli involving the presence or absence of a distinctive visual feature. Journal of the Experimental Analysis of Behavior, 1971, 16, 327-336.

Fleshle R, M., \& Hoffman, H. S. A progression for generating variable interval schedules. Journal of the Experimental Analysis of Behavior, 1962, 5, 529-530.

Grant, D. A., \& Schneider, D. E. Intensity of the conditioned stimulus and strength of conditioning. I. The conditioned eyelid response to light. Journal of Experimental Psychology, 1948. $38,690-696$.

Grant, D. A., \& Schneider, D. E. Intensity of the conditioned stimulus and strength of conditioning. II. The conditioned galvanic skin response to an auditory stimulus. Journal of Experimental Psychology, 1949, 39, 35-40.

Hearst, E. Some persistent problems in the analysis of conditioned inhibition. In R. A. Boakes \& M. S. Halliday (Eds.), Inhibition and learning. London: Academic Press, 1972.

Hearst, E. Stimulus relationships and feature selection in learning and behavior. In S. H. Hulse, H. Fowler, \& W. K. Honig (Eds.), Cognitive processes in animal behavior. Hillsdale, N.J: Erlbaum, 1978.

JENKINS, H. M. Noticing and responding in a discrimination based on a distinguishing element. Learning and Motivation, $1973,4,115 \cdot 137$.

Jenkins, H. M., \& Sainsbury, R. S. The development of stimulus control through differential reinforcement. In N. J. Mackintosh \& W. K. Honig (Eds.), Fundamental issues in associative learning. Halifax: Dalhousie University Press, 1969.

Jenkins, H. M., \& Sainsbury, R. S. Discrimination learning with the distinctive feature on positive or negative trials. In D. Mostofsky (Ed.), Attention: Contemporary theory and analysis. New York: Appleton-Century-Crofts, 1970.

Mackintosh, N. J. Epilogue: Issues and problems in associative learning. In N. J. Mackintosh \& W. K. Honig (Eds.), Fundamental issues in associative learning. Halifax: Dalhousie University Press, 1969.

Pavlov, I. P. Conditioned reflexes (G. V. Anrep, trans.). Oxford: Oxford University Press, 1927.

Rescorla, R. A. Pavlovian conditioned inhibition. Psychological Bulletin, 1969, 72, 77-94.

REsCoRLA, R. A., \& LoLordo, V. M. Inhibition of avoidance behavior. Journal of Comparative and Physiological Psychology, 1965, 59, 406-412.

(Received for publication November 7, 1978; revision accepted September 18, 1979.) 